\title{
A large $\mathrm{H}$ I cloud near the centre of the Virgo cluster
}

\author{
T. Oosterloo ${ }^{1}$ and J. van Gorkom ${ }^{2,3}$ \\ 1 Netherlands Foundation for Research in Astronomy, Postbus 2, 7990 AA, Dwingeloo, The Netherlands \\ e-mail: oosterloo@astron.nl \\ 2 Department of Astronomy, Columbia University, 550 West 120th Street, New York, NY 10027, USA \\ 3 Kapteyn Astronomical Institute, University of Groningen, Postbus 800, 9700 AV Groningen, The Netherlands
}

Received 22 April 2005 / Accepted 18 May 2005

\begin{abstract}
We report the discovery of a large H I cloud in the central regions of the Virgo cluster. It is $110 \times 25 \mathrm{kpc}$ in size and contains $3.4 \times 10^{8} M_{\odot}$ of $\mathrm{HI}$. The morphology and kinematics of this cloud strongly suggest that it consists of $\mathrm{H}$ I removed from the galaxy NGC 4388 by ram-pressure stripping. It is more likely the result of an interaction of the ISM of NGC 4388 with the hot halo of the M 86 group and not with the ICM centred on M 87. The large extent of the plume suggests that gas stripped from cluster galaxies can remain neutral for at least $10^{8} \mathrm{yr}$. Locally, the column density is well above $10^{20} \mathrm{~cm}^{-2}$, suggesting that the intra-cluster H II regions known to exist in Virgo may have formed from gas stripped from cluster galaxies. The existence of the H I plume suggests that stripping of infalling spirals contributes to the enrichment of the ICM. The H I object in the Virgo cluster recently reported by Minchin et al. (2005, ApJ, 622, L21) may have a similar origin and may therefore not be a "dark galaxy".
\end{abstract}

Key words. intergalactic medium - galaxies: interaction - galaxies: clusters: individual: Virgo cluster galaxies: individual: NGC 4388

\section{Introduction}

It is well known that clusters are hostile environments for galaxies and that galaxies in clusters differ in many aspects from field galaxies. Due to the high galaxy density, interactions between galaxies occur frequently, affecting and transforming them. Moreover, cluster galaxies move at high speed through the intra-cluster medium (ICM) and this ICM can remove gas from the galaxies by ram-pressure stripping. One very clear manifestation of these effects is that the properties of the neutral hydrogen of cluster spirals are severely affected by cluster related processes. Many cluster spirals contain (sometimes much) less neutral hydrogen in comparison to what expected for their type and luminosity (e.g. Cayatte et al. 1990; Solanes et al. 2001). H I imaging studies of such galaxies show that their H I disk is often truncated to well inside the optical disk (e.g. Warmels 1988; Cayatte et al. 1990).

The processes responsible for gas removal (tidal interactions and ram-pressure stripping by the ICM) have been studied in detail, both theoretically (e.g. Abadi et al. 1999; Quilis et al. 2000; Schulz \& Struck 2001; Vollmer et al. 2001) and observationally (e.g. Warmels 1988; Cayatte et al. 1990; Solanes et al. 2001; Kenney et al. 2004). Nevertheless, questions remain. One source of uncertainty is that the orbit of a galaxy through the cluster is unknown so that assumptions have to be made about where and when the stripping occurred. Even less clear is the fate of the gas once it has been removed from a cluster galaxy and has ended up in intra-cluster space. Being embedded in the hot cluster halo, one might expect the stripped gas to evaporate and enrich the cluster halo (e.g. Veilleux et al. 1999; Vollmer et al. 2001). However, the timescale for this evaporation is uncertain, e.g. because it depends on the structure of the magnetic field. Moreover, if the column density of the stripped gas is sufficiently high, it may condense into clouds of mostly molecular gas with only a skin of neutral hydrogen (Vollmer et al. 2001). About $10 \%$ of the stripped material would then be in atomic hydrogen, the rest in molecular gas.

There are only a few observational constraints on the fate of stripped gas and it is not clear what is more likely to happen under which circumstances: does the gas end up being very hot or very cold? Only one clear case is known of a trail of stripped gas in a cluster. In the cluster A1367, Gavazzi et al. (2001) have found a 75-kpc long trail of $\mathrm{H} \alpha$ emission emanating from two cluster galaxies, likely to be the result of a combination of tidal interaction and ram pressure. It is not known what ionises this trail and whether it has a neutral counterpart. Its extent suggests that the evaporation timescale is at least $10^{8} \mathrm{yr}$. Perhaps another clue is that some small, isolated intra-cluster $\mathrm{H}$ II regions exist in the Virgo cluster (Gerhard et al. 2002; Cortese et al. 2004). If these form from stripped gas, this would indicate that the cold scenario, at least sometimes, occurs.

A prime candidate for further study is the Virgo spiral NGC 4388. This galaxy is moving with a velocity of (at least) $1500 \mathrm{~km} \mathrm{~s}^{-1}$ through the Virgo cluster and is one of its most 
H I deficient galaxies (having lost $85 \%$ of its $\mathrm{H} \mathrm{I}$; Cayatte et al. 1990). Its H I disk is truncated to well within the optical disk (Cayatte et al. 1990). Moreover, the galaxy has a large plume of ionised gas, visible in $\mathrm{H} \alpha$, extending $35 \mathrm{kpc}$ off the galactic plane to the NE (Yoshida et al. 2002). X-ray observations show the existence of soft X-ray emission out to $16 \mathrm{kpc}$ (and possibly $30 \mathrm{kpc}$ ), in a similar location as the optically visible ionised gas (Iwasawa et al. 2003). Finally, by comparing single dish and VLA H I observations, Vollmer \& Huchtmeier (2003) find evidence for neutral gas out to at least $20 \mathrm{kpc}$ NE of NGC 4388 with a H I mass of $6 \times 10^{7} M_{\odot}$.

Taken together, these observations indicate that the ISM of NGC 4388 is strongly affected by the passage through the cluster and that there is large reservoir of gas out to at least $30 \mathrm{kpc}$ NE of NGC 4388. No agreement exists about what is responsible for the gas found NE of NGC 4388. Ram pressure has been suggested (Vollmer \& Huchtmeier 2003), but alternative explanations have also been proposed such as a nuclear outflow related to the Seyfert AGN in NGC 4388 (Veilleux et al. 1999) or accretion of a small companion (Yoshida et al. 2002). The optical- and X-ray data suggest that the plume closest to the galaxy is photo-ionised by the AGN residing in NGC 4388, but it is not clear what ionises the gas further out.

Here we present new $\mathrm{HI}$ observations of the region $\mathrm{NE}$ of NGC 4388. These observations show that the gas NE of NGC 4388 extends much further than previously thought. The data provide strong evidence that ram pressure pushed this gas from NGC 4388. Most likely it is not the ICM of the Virgo cluster itself, but the hot halo around M 86 that is stripping NGC 4388. Moreover, stripped neutral gas is able to survive for at least $10^{8} \mathrm{yr}$. Locally, the column density is possibly high enough for star formation to occur.

\section{The detection of a large $\mathrm{HI}$ plume NE of NGC 4388}

A region NE from NGC 4388 (pointing centre $\alpha: 12^{\mathrm{h}} 26^{\mathrm{m}} 00^{\mathrm{s}} \delta$ : $12^{\circ} 45^{\prime} 00^{\prime \prime}$, J2000) was observed for $12 \mathrm{hr}$ with the Westerbork Synthesis Radio Telescope (WSRT) on 26 February 2005. A $20-\mathrm{MHz}$ bandwidth was used, centred on a heliocentric velocity of $1500 \mathrm{~km} \mathrm{~s}^{-1}$, with 1024 channels and 2 independent polarisations. The standard WSRT 72-m configuration was used. The data were calibrated following the standard WSRT recipes using the MIRIAD software. The datacube was made with a channel width of $16.5 \mathrm{~km} \mathrm{~s}^{-1}$. Hanning smoothing was applied to the final datacube, giving a velocity resolution of $33.0 \mathrm{~km} \mathrm{~s}^{-1}$. Robust weighting was used with robustness = 0.5. Due to the low declination of the field and the E-W layout of the WSRT the beam is very elongated: $18.0^{\prime \prime} \times 95.1^{\prime \prime}\left(\mathrm{PA} 0^{\circ}\right)$. The noise in the datacube is $0.27 \mathrm{mJy}$ beam $^{-1}$. This gives a 3- $\sigma$ mass sensitivity of $1.6 \times 10^{6} M_{\odot}$ per resolution element (assuming a distance of $16 \mathrm{Mpc}$ ) and a 3- $\sigma$ column-density sensitivity of $1.7 \times 10^{19} \mathrm{~cm}^{-2}$. Cubes with lower spatial resolution were also made, but these did not reveal any new features. Hence, only the full-resolution data are discussed.

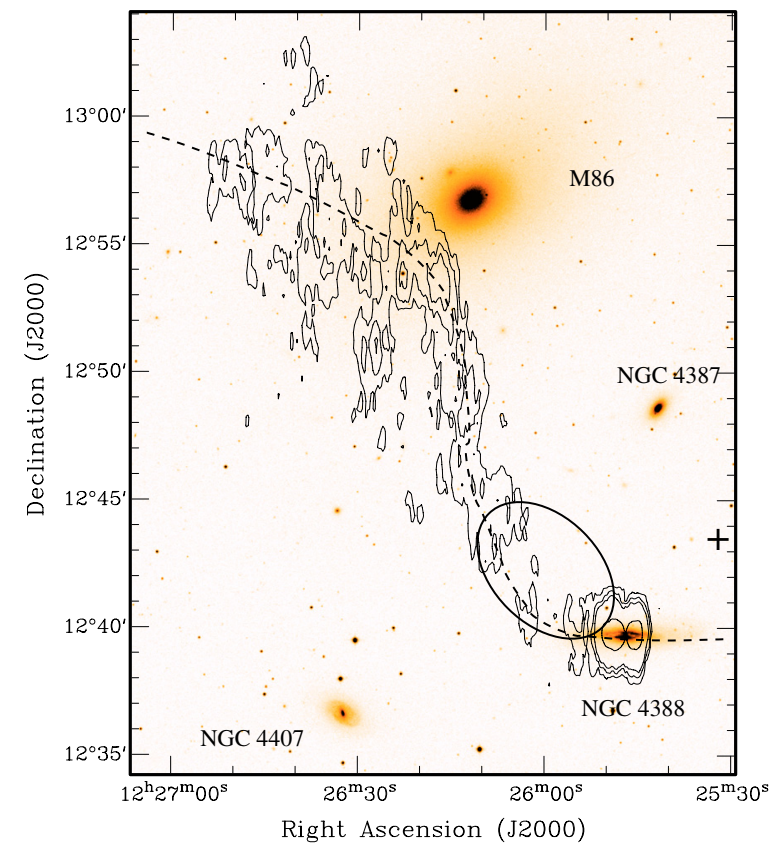

Fig. 1. Integrated $\mathrm{H}$ I emission of the plume. Contour levels are 1.0, $5.0,10.0$ and 50.0 in units of $10^{19} \mathrm{~cm}^{-2}$. The locus along which the position-velocity plot of Fig. 2 is taken is indicated. The ellipse indicates where the ionised gas is detected (Yoshida et al. 2002), while the plus sign gives the location of the $\mathrm{H}$ II region found by Gerhard et al. (2002).

\subsection{Properties of the plume}

In Fig. 1 the integrated $\mathrm{H}$ I emission of the observed region is given, superposed on an optical image taken from the Digitized Sky Survey. The integrated H I image was made by selecting by hand those regions in the datacube containing $\mathrm{HI}$ emission. This figure shows that we detect a large, linear plume of $\mathrm{HI}$, starting from the E side of NGC 4388 and going in a NE direction. The velocity range of the plume is roughly $2000-2550 \mathrm{~km} \mathrm{~s}^{-1}$ which is close to the systemic velocity of NGC $4388\left(2524 \mathrm{~km} \mathrm{~s}^{-1}\right)$. In Fig. 2 a position-velocity plot is given taken along the ridge of maximum flux density of the plume (indicated in Fig. 1). The fact that the plume connects to NGC 4388 not only on the sky but also in velocity demonstrates that the plume is physically related to NGC 4388.

Given the pointings used in the single-dish H I observations of Vollmer \& Huchtmeier (2003), it is clear that they detected the southern part of the plume. Interestingly, Davies et al. (2004) have detected the H I plume in their survey of the Virgo cluster (their VIRGOHI4), however they interpreted this emission as belonging to a galaxy behind M 86 and hidden from our view. Quite importantly, the H I plume appears to be the neutral extension of the plume of ionised gas detected by Yoshida et al. (2002) and Iwasawa et al. (2003). The ionised and the neutral filaments clearly form one structure.

The extent of the plume is roughly 110 by $25 \mathrm{kpc}$ and its H I mass is $3.4 \times 10^{8} M_{\odot}$. This is about the same amount of neutral hydrogen as we detect in NGC $4388\left(3.6 \times 10^{8} M_{\odot}\right)$. The peak of the H I emission is not located near NGC 4388 but instead is close to M 86, about $10 \mathrm{kpc}$ SSE of this galaxy. 


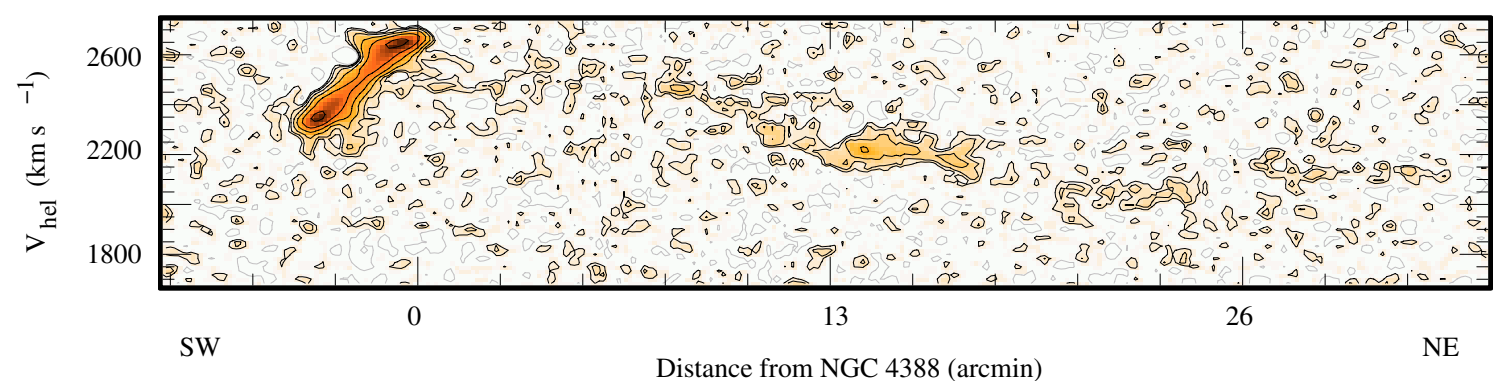

Fig. 2. Position-velocity plot of the plume taken along the locus shown in Fig. 1 . Contour levels are $-0.35,+0.35,0.7,1.4,2.8, \ldots \mathrm{mJy}$ beam ${ }^{-1}$.

At this location, the observed column densities reach values just above $10^{20} \mathrm{~cm}^{-2}$. Given the large beam of our observations, the column density will locally exceed this value by a significant factor. Near M 86 the plume is also widest with a width of about $25 \mathrm{kpc}$. Going from M 86 towards NGC 4388, the column densities slowly decrease and near NGC 4388 the plume is barely detected at levels of $10^{19} \mathrm{~cm}^{-2}$. The distance from the maximum to NGC 4388 is about $70 \mathrm{kpc}$. The plume extends for another $40 \mathrm{kpc}$ to the NE from the location of the maximum. It is possible that the plume extends even further in this direction. The tip of the plume is about $19^{\prime}$ away from the pointing centre of the observations so that primary beam effects are significant there (the FWHM of the WSRT primary beam is $35^{\prime}$ ).

The position-velocity plot shows that the velocity of the plume slowly decreases from $2550 \mathrm{~km} \mathrm{~s}^{-1}$, i.e. the velocity of the eastern side of NGC 4388 , to about $2000 \mathrm{~km} \mathrm{~s}^{-1}$ at the end of the plume. The velocity width is about $100 \mathrm{~km} \mathrm{~s}^{-1}$, except in the region near the maximum where the velocity width is about $200 \mathrm{~km} \mathrm{~s}^{-1}$.

\section{Discussion}

\subsection{Origin}

The morphology and kinematics of the plume clearly suggest that it is gas pushed or pulled from NGC 4388, either by tidal interaction and/or ram pressure stripping. The relative velocities between galaxies in the Virgo cluster are high, causing tidal interactions between galaxies to occur frequently, but to be short in duration. The cumulative effect of many tidal interactions (harassment; Moore et al. 1998) can be significant, but one may expect that harassment would not produce a single, long linear filament such as the H I plume, but instead a more diffuse or chaotic structure.

An alternative is that the interaction is with a larger scale mass such as with a cluster sub-clump. Such interactions more likely produce long, linear filaments because the timescale is longer. A nice example is a long stellar trail in the Centaurus cluster. This trail is likely the result of a tidal interaction of NGC 4709 with substructure of the Centaurus cluster (Calcáneo-Roldán et al. 2000). The plume near NGC 4388 bears a striking morphological resemblance to this stellar trail in the Centaurus cluster. However, for this kind of interactions to produce large tails, the interaction has to be prograde (Calcáneo-Roldán et al. 2000), i.e. the internal spin vector of NGC 4388 has to be more or less parallel to that of the orbit of NGC 4388 relative to the substructure it is interacting with. However, the sense of rotation of NGC 4388 is such that, given the position and the large positive velocity of NGC 4388, any large-scale tidal interaction is retrograde, whether the interaction is with the mass centred on M 87, or the M 86 sub-clump.

The main argument, however, against a tidal origin is that a deep optical image (Phillips \& Malin 1982) shows that a stellar counterpart is completely absent. In general, given its larger extent, the H I disk of a galaxy is more susceptible to tidal distortions than the stellar disk. However, given that the H I disk of NGC 4388 is affected to well within the optical disk, one would expect at least some stellar counterpart to the plume. The deep optical image does show very diffuse stellar emission all around M 86 and NGC 4388, suggesting that tidal interactions have occurred several times in the past. Moreover, the image also shows a small stellar feature going SW from NGC 4388. This suggests that NGC 4388 may have experienced minor tidal effects recently. These will not have caused the plume, but they may shaken the ISM of NGC 4388 such that other effects, such as ram pressure, have had a more dramatic impact.

The lack of a stellar counterpart to the H I plume, and the $\mathrm{H}$ I disk being truncated to well inside the optical disk, argue in favour of a ram pressure related origin. Indeed, NGC 4388 is often discussed as a good example of a galaxy undergoing rampressure stripping (Vollmer et al. 2001; Vollmer \& Huchtmeier 2003). A fairly detailed model for the case where NGC 4388 is interacting with the halo of hot gas centred on M 87 is given by Vollmer \& Huchtmeier (2003). The observed morphology and kinematics of the plume match those predicted by this model quite well (compare e.g. their Fig. 5 with our Fig. 2). However, another possibility is that the HI plume is due to stripping by the halo of M 86. Projection effects make an unambiguous association impossible, but the plume appears to be much closer to M 86 (10 kpc projected distance) than to M 87 (350 kpc projected distance). The density distribution along the H I plume is what one would expect from an interaction with M 86. The vicinity of the maximum density near M 86, the fact that the plume seems broadest there and the large profile widths, may suggest the ram-pressure effects were strongest near M 86. The kinematics of the plume is well explained by the model of Vollmer \& Huchtmeier (2003), i.e. going from NGC 4388 along the plume, the velocities become less positive. However, the main reason for this is that in the model NGC 4388 has a positive velocity w.r.t. the ICM assumed to 
do the stripping. Given that M 86 has a negative velocity w.r.t. NGC 4388, similar gas kinematics would result from an interaction with the halo of M 86. Detailed simulations would be needed to distinguish between the two possibilities.

According to Finoguenov et al. (2004), the halo of M 86 can provide the same ram pressure as the halo of $\mathrm{M} 87$, so a passage of NGC 4388 close to M 86 will have similar effects as in the model of Vollmer \& Huchtmeier (2003). In the model of Vollmer \& Huchtmeier (2003) for the interaction with M 87, the maximum pressure used is $5000 \mathrm{~cm}^{-3}\left(\mathrm{~km} \mathrm{~s}^{-1}\right)^{2}$. The relative velocity of NGC 4388 with M 86 is much higher than with M 87, so the implied ICM density is lower: $4 \times 10^{-4} \mathrm{~cm}^{-3}$ vs. $1.25 \times 10^{-3} \mathrm{~cm}^{-3}$. The current X-ray data do not allow to estimate to which radius of the M 86 halo this corresponds.

\subsection{Fate of gas}

Different predictions are made in the literature for what happens to the gas once it has been stripped. The large extent of the $\mathrm{H}$ I indicates that it can survive for a fairly long time. Although projection factors are unknown, a rough estimate of the age of the plume can be made. Assuming NGC 4388 is moving in the plane of the sky with a velocity of $500 \mathrm{~km} \mathrm{~s}^{-1}$, the length of the plume implies an age of a few times $10^{8} \mathrm{yr}$. This may suggest that evaporation by the ICM is slow, e.g. because the heat flow is saturated and/or that a tangled magnetic field slows down the heat flow into the plume (Vollmer et al. 2001). The X-ray halo of M 86 is much smaller in size than that of M 87 so the ICM density drops faster with radius, while also the higher relative velocity of NGC 4388 with M 86 implies lower densities for the region responsible for stripping NGC 4388. This could mean that if the plume is due to stripping by M 86, longer evaporation times would then be expected. Given the long evaporation timescale, dense clumps may form in the plume and part of the stripped ISM may become molecular. Vollmer et al. (2001) predict that in such a scenario only $10 \%$ of the total gas mass may be in the form of neutral hydrogen. In this case, the total gas mass of the plume is $3.4 \times 10^{9} \mathrm{M}_{\odot}$.

In and around normal spirals, if the H I column density is above a few times $10^{20} \mathrm{~cm}^{-2}$, star formation almost invariably occurs (Schaye 2004). The column densities in the plume likely exceed this threshold in some locations, in particular near M 86. Hence, star formation could occur locally in the plume, provided the processes that regulate star formation for a cloud in the ICM are similar to those for gas clouds in spiral galaxies. Indeed, at least 17 extragalactic $\mathrm{H}$ II regions exist near M 86 and NGC 4388 (Gerhard et al. 2002; Cortese et al. 2004). We do not detect $\mathrm{H}$ I near the H II region found close to NGC 4388 by Gerhard et al. (2002) so there may not be a direct connection for this particular H II region. The number of intra-cluster $\mathrm{H}$ II regions is small, so most of the mass of objects such as the plume will end up being part of the hot ICM and will enrich it.

An interesting question is how common neutral gas clouds, as the one we observe near NGC 4388, are. There are many Virgo spirals currently undergoing ram-pressure stripping. If the stripped gas remains observable for at least $10^{8} \mathrm{yr}$, more plumes should exist. Many Virgo spirals have been imaged in $\mathrm{HI}$ and no large plumes have been reported. However, our data show that new, deeper observations may reveal plumes in places where it is thought they do not exist. Other recent observations also start to show much more detail in the $\mathrm{H} \mathrm{I}$ distribution of stripped galaxies (e.g. Chung et al. 2005). Moreover, in a blind single-dish survey of the Virgo cluster, Davies et al. (2004) found 3 H I clouds (of which the plume of NGC 4388 turns out to be one) that do not have an optical counterpart. So it appears that a few more H I plumes may exist in the Virgo cluster. Minchin et al. (2005) claim that one of the clouds found by Davies et al. (2004) could be a "dark galaxy", i.e. an object containing gas and dark matter, but no stars. However, located about $200 \mathrm{kpc}$ from this cloud is the galaxy NGC 4254, a spiral that shows signs of both tidal interaction and ram-pressure stripping (Phookun et al. 1993). The velocity of the gas cloud and NGC 4254 are very similar, so it is quite possible that this cloud has a similar origin as the plume near NGC 4388.

Acknowledgements. We would like to thank Bernd Vollmer for his valuable input. The WSRT is operated by the Netherlands Foundation for Research in Astronomy (ASTRON) with the support from the Netherlands Foundation for Scientific Research (NWO). This research has made use of the NASA Extragalactic Database (NED). The Digitized Sky Survey was produced at the Space Telescope Science Institute under US Government grant NAG W-2166.

\section{References}

Abadi, M. G., Moore, B., \& Bower, R. G. 1999, MNRAS, 308, 947

Calcáneo-Roldán, C., Moore, B., Bland-Hawthorn, J., Malin, D., \& Sadler, E. M. 2000, MNRAS, 314, 324

Cayatte, V., van Gorkom, J. H., Balkowski, C., \& Kotanyi, C. 1990, AJ, 100, 604

Chung, A, van Gorkom, J. H., Kenney, J. D. P., \& Vollmer, B. 2005, in Extraplanar Gas, ed. R. Braun, ASP Conf. Ser., 331, 275

Cortese, L., Gavazzi, G., Boselli, A., \& Iglesias-Parama, J. 1990, A\&A, 416, 119

Davies, J., Minchin, R., Sabatini, S., et al. 2004, MNRAS, 349, 922

Finoguenov, A., Pietsch, W., Aschenbach, B., \& Miniati, F. 2004, A\&A, 415,415

Gavazzi, G., Boselli, A., Mayer, L., et al. 2001, ApJ, 563, L23

Gerhard, O., Arnaboldi, M., Freeman, K. C., \& Okamura, S. 2002, ApJ, 580, L121

Iwasawa, K., Wilson, A. S., Fabian, A. C., \& Young, A. J. 2003, MNRAS, 345, 369

Kenney, J. D. P., van Gorkom, J. H., \& Vollmer, B. 2004, AJ, 127, 3361

Minchin, R., Davies, J., Disney, M., et al. 2005, ApJ, 622, L21

Moore, B., Lake, G., \& Katz, N. 1998, ApJ, 495, 139

Phillips, M. M., \& Malin, D. F. 1982, MNRAS, 199, 905

Phookun, B., Vogel, S. N., \& Mundy, L. G. 1993, ApJ, 418, 113

Quilis, Q., Moore, B., \& Bower, R. 2000, Science, 288, 117

Schaye, J. 2004, ApJ, 609, 667

Schulz, S., \& Struck, C. 2001, MNRAS, 328, 185

Solanes, J. M., Manrique, A., González-Casado, G., et al. 2001, AJ, 124,2440

Veilleux, S., Bland-Hawthorn, J., Cecil, G., Tully, R. B., \& Miller, S. T. 1999, ApJ, 520, 111

Vollmer, B., Cayatte, V., Balkowski, C., \& Duschl, W. 2001, ApJ, 561, 708

Vollmer, B., \& Huchtmeier, W. 2003, A\&A, 406, 427

Warmels, R. H. 1988, A\&AS, 72, 57

Yoshida, M., Yagi, M., Okamura, S., et al. 2002, ApJ, 567, 118 